\title{
Laser Treatment of Epistaxis and Oral Bleeding in Hereditary Hemorrhagic Telangiectasia
}

\author{
Dan Yanivi,2, Uri Alkan1,2, Eitan Yaniv², Călin Bumbuluț ${ }^{3 *}{ }^{\circledR}$, Lea Rath-Wolfson ${ }^{2,4}$ \\ ${ }^{1}$ Department of Otorhinolaryngology-Head and Neck Surgery, Rabin Medical Center, Beilinson Hospital, Petach Tikva, Israel \\ ${ }^{2}$ Sackler Faculty of Medicine, Tel Aviv University, Tel Aviv, Israel \\ ${ }^{3}$ Civil Medical Society, Satu Mare, Romania \\ ${ }^{4}$ Department of Pathology, Rabin Medical Center, Hasharon Hospital, Petach Tikva, Israel \\ Email: ^bumbulutcalin@yahoo.com
}

How to cite this paper: Yaniv, D., Alkan, U., Yaniv, E., Bumbuluț, C. and Rath-Wolfson, L. (2019) Laser Treatment of Epistaxis and Oral Bleeding in Hereditary Hemorrhagic Telangiectasia. International Journal of Otolaryngology and Head \& Neck Surgery, 8, 180-190.

https://doi.org/10.4236/ijohns.2019.85020

Received: June 6, 2019

Accepted: August 30, 2019

Published: September 3, 2019

Copyright () 2019 by author(s) and Scientific Research Publishing Inc. This work is licensed under the Creative Commons Attribution International License (CC BY 4.0).

http://creativecommons.org/licenses/by/4.0/

\section{(c) (i) Open Access}

\begin{abstract}
Background: Hereditary hemorrhagic telangiectasia (HHT) is a relatively common, albeit under-recognized autosomal-dominant multisystemic vascular disorder. Epistaxis due to telangiectases in the nasal mucosa is the most common and often the earliest symptom of HHT. As many as $90 \%$ of affected individuals eventually experience recurrent epistaxis, with a mean frequency of 18 episodes per month. Prompted by the limitations of invasive treatment, researchers have directed attention to laser photocoagulation as an alternative nonsurgical treatment modality with promising results. Purpose: The purpose of the present study was to describe our experience with the state-of-the-art $980 \mathrm{~nm}$ diode laser for the treatment of bleeding lesions of the skin, nasal and oral mucosa in patients with HHT. Methodology/Principal: We treated 16 HHT patients with intractable bleeding from telangiectasias using the $980-\mathrm{nm}$ diode laser as an office procedure using local anesthesia. We recorded hemoglobin levels before and after treatment and used disability questionnaires. Results: All patients treated had an improvement in hemoglobin levels and disability scores. In patients with multiple lesions hemoglobin levels improved from a mean of 8.4 to $11.2(\mathrm{p}=0.008)$. The disability index in this group improved from a mean of 5.3 to $2.8(\mathrm{p}=0.007)$. Follow-up ranged between 4 and 12 months. Conclusions: The $980-\mathrm{nm}$ diode laser is a good office based solution for bleeding in HHT.
\end{abstract}

\section{Keywords}

HHT, Laser, Epistaxis, Osler-Weber-Rendu

\section{Introduction}

Hereditary hemorrhagic telangiectasia (HHT), also known as Osler-Weber-Rendu 
syndrome, is a relatively common, albeit under-recognized autosomal-dominant multisystemic vascular disorder. It affects all ethnic and racial groups and has a wide geographic distribution, with an overall incidence of 1 per 5000 to 10,000 persons [1] [2] [3]. The Curaçao criteria [4] for diagnosis of HHT include the following 4 criteria: Spontaneous recurrent epistaxis, multiple telangiectasias in typical locations (nose, lips, oral cavity, and skin), proven visceral arteriovenous malformations (AVM's) (lung, liver, brain, and spine) and first-degree family member with HHT. If three or four criteria are present, a patient has "definite HHT", while two gives "possible HHT". De novo mutations are rare. Penetrance approaches $100 \%$ by age 40 years.

The underlying vascular angiodysplasia is responsible for the two main manifestations of the disease: telangiectasia and arteriovenous malformations [1] [2] [3]. Telangiectases are small arteriovenous shunts involving dilated arterioles and venules. They appear as red spots of $1-2 \mathrm{~mm}$ diameter on the skin. In an electron microscopy study of HHT, Braveman et al. hypothesized that the earliest clinically detectable lesion of HHT is a focal dilatation of postcapillary venules. Over time, the venules continue to enlarge and eventually connect with dilated arterioles through capillaries [5]. As the vascular lesion increases in size, the capillary segments disappear, and a direct arterio-venous communication is formed. In fully developed telangiectases, the venules are markedly dilated and convoluted, extend through the entire dermis, and have excessive layers of smooth muscle without elastic fibers. However, few reports have provided direct evidence of the actual morphogenic evolution of cutaneous telangiectatic lesions. Ultrastructural studies have so far been performed only on skin samples, and there are no available histological data on mucosal (oral and nasal) telangiectases, despite their high clinical significance. AVMs, the second most prominent lesion of HHT, lack capillaries. They consist of direct connections between arteries and veins and are larger than telangiectases. It is these vascular abnormalities that lead to the common presenting symptoms of patients with HHT.

Most telangiectases in HHT are found in the oral, nasal, and gastrointestinal mucosa and the fingertips, whereas AVMs occur most commonly in the lungs, liver, and central nervous system [6] [7]. Epistaxis due to telangiectases in the nasal mucosa is the most common and often the earliest symptom of HHT. As many as $90 \%$ of affected individuals eventually experience recurrent epistaxis, with a mean frequency of 18 episodes per month. Some patients have severe epistaxis which can lead to chronic anemia, and others have mild nosebleeds that do not require treatment. In general, the frequency and severity of the epistaxis increase with advancing age, although there are patients who report no particular change in episodes over time, and some even show improvement [8] [9].

The neoV980 is an all-purpose surgical tool for a wide scope of clinical applications. The $980 \mathrm{~nm}$ wavelength offering almost equal absorption in water and blood, coupled with the higher power output of the neoV platform, enables safe and effective treatment of many conditions, from ear, nose and throat (ENT) and gynecological surgery. 
The neoV980 can deliver up to $20 \mathrm{~W}$ of output power from the fiber tip. Delivering high power energy through precision instruments and fiber optics, the unit provides sufficient power for a wide array of high value applications, enabling safe, precise, and efficient use [10].

As a result, the neoV980 can be used efficiently for precision cutting as well as coagulation of vessels for hemostasis. The aim of the present study was to describe our experience with the state-of-the-art $980 \mathrm{~nm}$ diode laser for the treatment of bleeding lesions of the skin, nasal and oral mucosa in patients with HHT.

\section{Materials and Methods}

\subsection{Patients and Setting}

The study group consisted of 16 patients with the diagnosis of HHT with recurrent epistaxis causing a detrimental effect on quality of life who attended a single university-affiliated, tertiary, medical center from June 2014 to October 2015. The inclusion criteria were: Patients with a diagnosis of HHT according to $\mathrm{Cu}$ raçao criteria, Epistaxis Severity Score (ESS) [11] score over 4 (ESS 1 - 4 correlates with mild severity, $4-7$ moderate severity and $8-10$ is severe disease).

Exclusion criteria included: patients with an ESS score smaller than 4, patients with less than 3 criteria of the Curaçao criteria and pediatric patients.

All were treated with coagulation using the neoV $980-\mathrm{nm}$ diode laser (neoLaser, Caesarea, Israel).

Hemoglobin levels and ESS scores were measured before treatment and 12 weeks after treatment.

\subsection{Laser Procedure}

Laser photocoagulation was performed as an office procedure under local anesthesia. For nasal lesions, anesthesia was achieved with a nasal spray of lidocaine $2 \%$ and adrenaline 1:20,000; for lesions of the skin and oral cavity mucosa, anesthesia was achieved with infiltration with lidocaine $2 \%$ and adrenaline $1: 100,000$. Patients were treated in continuous wave (CW) mode, with power levels starting from $12 \mathrm{~W}$. The optic fiber was maintained at a distance of $3-5$ $\mathrm{mm}$ from the lesion throughout. Laser shots were controlled by activating a foot pedal. Protective goggles were provided to both patient and treating physician.

The laser transmits up to 20 watts of power, either continuous or pulsed, through a flexible optical fiber consisting of a 400-micron silica core, 440-micron silica cladding with 465-micron Polyimide coating and a bare tip, measuring a length of 2.6 meters, with a numerical aperture of 0.22 at the tip. The laser may be applied with and without contact of the fiber with the tissue. The thin optical fibers are highly amenable for use inside the nasal cavity (Figure 1).

\subsection{Self-Report Questionnaire}

Patients were asked to complete the ESS ([11], see Appendix) at the clinic visits 


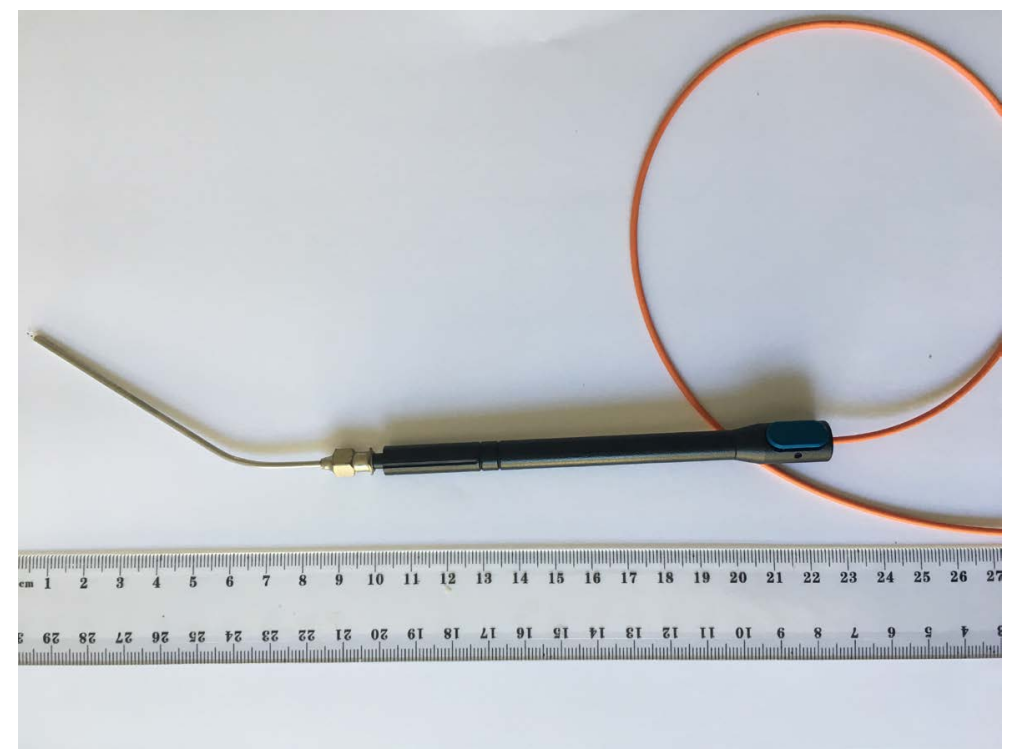

Figure 1. The hand piece and silica fiber.

just before and after treatment. The instrument measures the severity of epistaxis in the previous month by six factors associated with self-reported severity, including: frequency, duration, intensity, need for medical attention, anemia, and need for transfusion. The normalized ESS is within a range of 0 (no epistaxis) and 10 (most severe epistaxis).

\subsection{Outcome Measures}

The effect of laser photocoagulation was measured by the change in hemoglobin levels and ESS scores from before to after treatment.

\subsection{Statistical Analysis}

Data are presented as mean \pm standard deviation. The Wilcoxon signed rank sum test was used to analyze differences in the objective and subjective measure before and after treatment. All reported $\mathrm{p}$ values are two-sided; $\mathrm{p}<0.05$ was considered statistically significant. For the statistical analyses, we used SPSS 15.01.1 software (SPSS Inc. Chicago, IL, USA).

\subsection{Ethical Considerations}

All patients signed an informed consent before the laser procedure.

\section{Results}

Sixteen patients (10 female, 6 male) aged 29 to 76 years (average 56.6) met the inclusion criteria.

Lesion sites where the nasal mucosa in 9 patients, the nose and facial skin in 2 , and the oral cavity in 5 .

All patients were hemodynamically stable at the time of the laser procedure. Ten patients required one session of laser photocoagulation and 6 patients re- 
quired two sessions, 2 - 6 weeks apart. The duration of follow-up ranged from 4 to 12 months.

Seven patients had a single bleeding lesion at the time of treatment (Figure 2, Figure 3). All of these patients showed complete cessation of bleeding after treatment. Their mean hemoglobin level was $8.9 \pm 1.8 \mathrm{~g} / \mathrm{dL}$ before treatment and $12.1 \pm 1 \mathrm{~g} / \mathrm{dL}$ after $(\mathrm{p}=0.018)$. Corresponding mean severity of the ESS scores were $5.0 \pm 1$ before treatment and 0 after the treatment $(p=0.018)$.

Nine patients had multiple lesions at the time of treatment. In this group, we treated the most prominent lesions ( $1-4$ at each session) (Figure 4, Figure 5).

Patients reported a marked improvement in the intensity of bleeding and in the frequency and severity of epistaxis, with longer bleeding-free periods. Mean hemoglobin level in this group was $8.4 \pm 3.8 \mathrm{~g} / \mathrm{dL}$ before treatment and $11.2 \pm 1$ $\mathrm{g} / \mathrm{dL}$ after (in patients needing two treatment hemoglobin levels recorded after second treatment) $(\mathrm{p}=0.008)$, and mean ESS severity scores were $5.33 \pm 1.6$ and $2.8 \pm 1.1$ respectively $(\mathrm{p}=0.007)$.

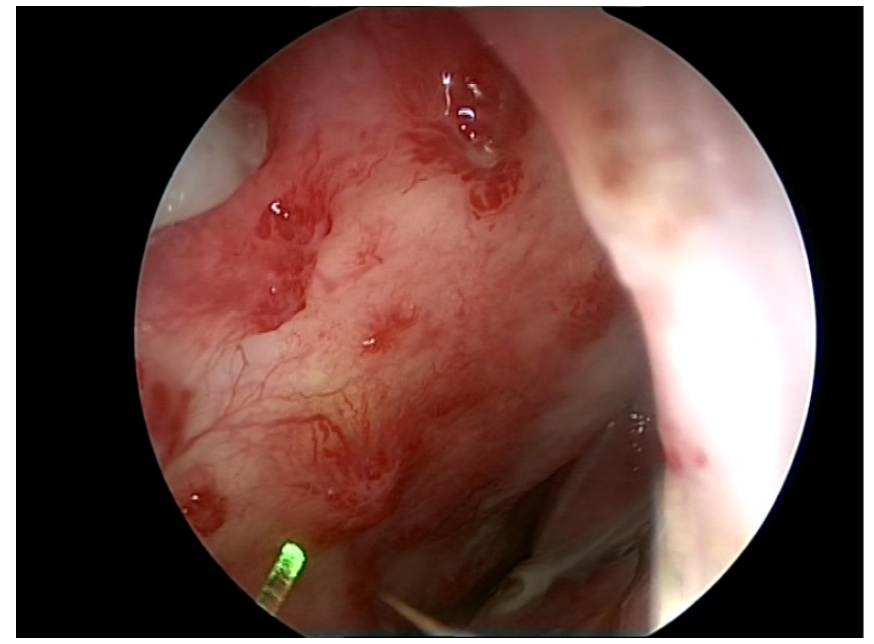

Figure 2. Telangiectatic skin lesion at the opening of the right nostril before treatment is seen in the center.

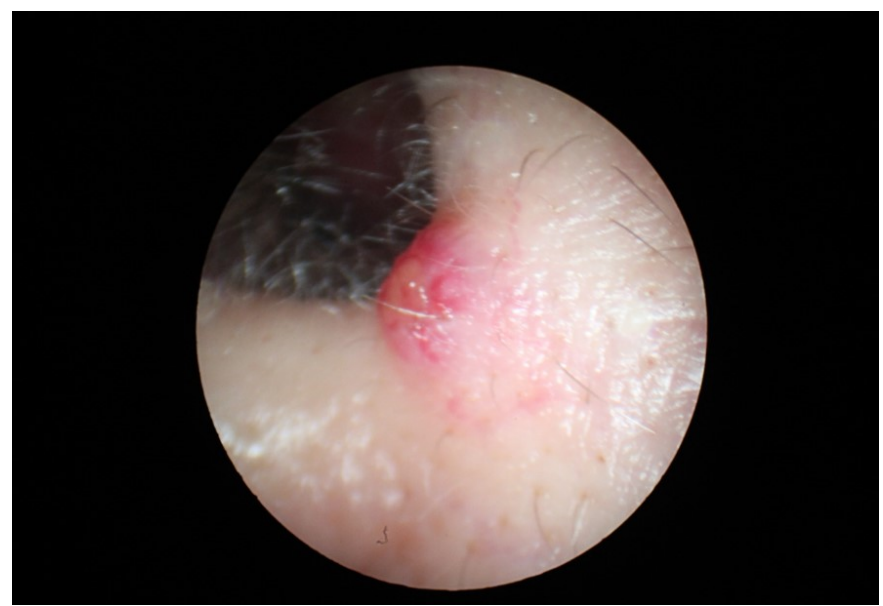

Figure 3. The same lesion immediately after treatment. 


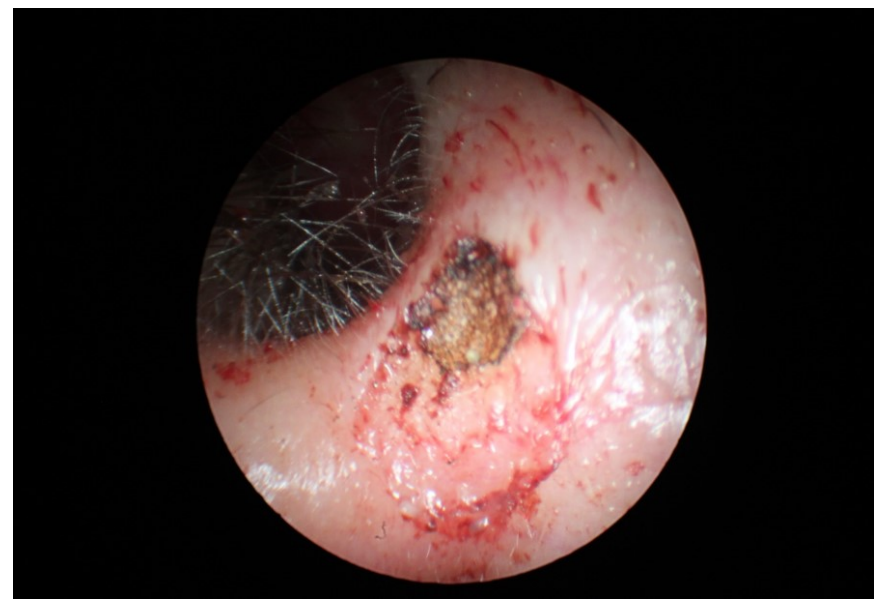

Figure 4. Multiple teleangiectatic nasal lesions before treatment.

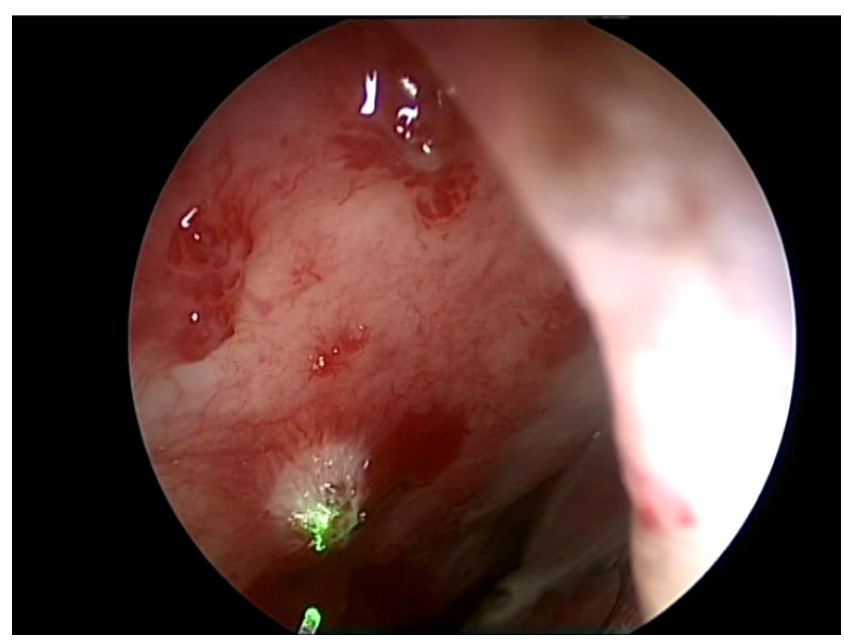

Figure 5. One of these lesions right after the treatment.

There were no recurrences in the treated lesions in either group during follow-up.

\section{Discussion}

The treatment of epistaxis consists of local measures, including application of local pressure and ice, combined with high-dose antifibrinolytic agents, such as Tranexamic acid, administered either locally, orally (1 - $2 \mathrm{~g} 3 \mathrm{x}$ daily) or intravenously at the time of epistaxis [12]. Systemic antiestrogen drugs such as tamoxifen have also proven very effective in most patients, but usage is limited in women of reproductive age [13]. In the presence of anemia, patients may require oral iron supplementation or, rarely, parenteral iron therapy with or without blood transfusion, depending on the level of anemia and clinical symptoms. If conservative measures are insufficient and the frequency and duration of episodes impair the patient's quality of life, chemical and electrocoagulation may be used. However, while these modalities are tolerable as a single treatment or for a restricted area inside the nasal cavity, more extensive use poses a risk of wide- 
spread scarring and damage to the cilia and mucous glands with crust formation, leading to compromised function. Embolization of the external carotid artery branches is effective for short-term relief of symptoms, but not for long-term management. It also might lead to ischemia-related complications [14]. In very severe cases closure of the nostrils is a good treatment, but it leads to complete dysfunction of the nose [15].

Prompted by the limitations of invasive treatment, researchers have directed attention to laser photocoagulation as an alternative nonsurgical treatment modality with promising results [15] [16].

Laser ablation of nasal telangiectasias has thus gained favor in managing these patients in a safe and effective manner and offers the additional advantage of being performed on an outpatient basis. It is worthwhile to note that its benefits appear to last for only a few months, often necessitating repeated treatments. Nevertheless, it is not surprising that effective control of epistaxis episodes is associated with improved quality of life in HHT patients [17].

The most common symptom of HHT is recurrent epistaxis (up to $90 \%$ of patients), which may lead to a decrease in quality of life, hospitalizations, and the need for multiple blood transfusions [8] [9]. Oral cavity bleeding is also very common and has a significant effect on quality of life. Ideally, bleeding should be treated locally with minimum side effects and without mucosal and glandular damage. Laser photocoagulation is a potentially superior method of treatment than chemical and electrocoagulation because it avoids the risk of large areas of scar tissue and nasal stricture or vascular damage. Popular devices include the KTP laser $(532 \mathrm{~nm})$, the pulse dye laser $(585 \mathrm{~nm})$, and various diode lasers at the near infrared range (780 - $1064 \mathrm{~nm})$.

The present study evaluated the effect of the neoV 980-nm diode laser. The $980 \mathrm{~nm}$ wavelength is selectively absorbed by hemoglobin and minimally absorbed in water, allowing for its transmission through the epithelium and coagulation of telangiectasis of the nasal mucosa, without damaging the vessel wall or mucosal-coating epithelium [18]. Because it penetrates more deeply into tissue, the neoV laser may yield better results than lower-wavelength lasers when larger vessels located a few millimeters below the mucosal surface are involved [19].

The good long-term clinical outcome of the laser-treated patients with HHT in the present study suggests the absence of a clinically significant adverse effect of the laser treatment on mucosal or glandular tissue. As the mucosa is undamaged, repeated laser sessions at minimal intervals may be used if needed without compromising nasal function.

A study by Karapantzos et al. utilizing the International Quality of Life Assessment Questionnaire corroborated these findings, indicating that laser treatment for HHT-related epistaxis produces long-lasting significant improvement in quality of life and mental health outcomes [20]. In contrast, Jørgensen et al. reported that there was no significant difference in quality of life using the SF-36 before and 6 months after laser treatment despite decreased epistaxis severity [21].

A division of the patients in our study by number of lesions affected (one vs 
multiple on endoscopic examination) yielded interesting findings. Patients with one lesion had an excellent outcome, with complete cessation of bleeding after the procedure and throughout follow-up (4 - 12 months). Their mean hemoglobin level increased by $3.2 \mathrm{~g} / \mathrm{dL}$, and their severity score improved by 4.0 points. These patients appear to be ideal candidates for local treatment with the $980 \mathrm{~nm}$ diode laser and can be spared systemic treatment with its possible side effects.

In the patients with multiple lesions, results were satisfactory. All showed a marked improvement in quality of life, with a decrease in bleeding. Mean hemoglobin increased by $2.8 \mathrm{~g} / \mathrm{dL}$ and severity score improved by 2.5 points.

Limitations of the study include a small sample size and short follow-up. Sample size included all patients with retractable bleeding that met the study criteria during the study period. As for the short follow-up, the study clearly shows an improvement in symptoms and quality of life for the short term, but due to the nature of the disease, it is almost certain that these patients will have new lesions and epistaxis episode during their lifetime. The fact that the laser does not scar the mucosa significantly allows for repeat treatments if necessary.

\section{Conclusion}

In conclusion, the neoV $980-\mathrm{mm}$ laser is an excellent treatment option for the treatment of HHT-associated epistaxis, with good results and no side effects. The procedure can be done in the office. Its use allows physicians to reserve systemic treatment for more complicated cases.

\section{Ethics Approval and Consent to Participate}

The research study was approved by the local ethics committee, a consent form detailing all the relevant information regarding the study procedures was conceived and signed by the subjects who agreed to participate.

\section{Consent for Publication}

The informed consent and agreement to participate in the study was obtained in all cases.

\section{Availability of Data and Materials}

The datasets used and/or analyzed during the current study are available from the corresponding author on reasonable request.

\section{Funding}

This research did not receive any specific grant from funding agencies in the public, commercial, or not-for-profit sectors.

\section{Authors' Contributions}

DY, UA, EY, CB, and LRW analyzed and interpreted the patient data regarding 
the Laser treatment of Epistaxis and Oral Bleeding in Hereditary Hemorrhagic Telangiectasia, with equal contributions in writing the manuscript. All authors read and approved the final manuscript.

\section{Conflicts of Interest}

The authors declare that they have no competing interests.

\section{References}

[1] Peery, W.H. (1987) Clinical Spectrum of Hereditary Hemorrhagic Telangiectasia (Osler-Weber-Rendu Disease). The American Journal of Medicine, 82, 989-997. https://doi.org/10.1016/0002-9343(87)90162-8

[2] Guttmacher, A.E., Marchuk, D.A. and White, R.I. (1995) Hereditary Hemorrhagic Telangiectasia. The New England Journal of Medicine, 333, 918-924. https://doi.org/10.1056/NEJM199510053331407

[3] Kjeldsen, A.D., Vase, P. and Green, A. (1999) Hereditary Haemorrhagic Telangiectasia: A Population-Based Study of Prevalence and Mortality in Danish Patients. Journal of Internal Medicine, 245, 31-39. https://doi.org/10.1046/j.1365-2796.1999.00398.x

[4] Shovlin, C.L., Guttmacher, A.E., Buscarini, E., et al. (2000) Diagnostic Criteria for Hereditary Hemorrhagic Telangiectasia (Rendu-Osler-Weber Syndrome). American Journal of Medical Genetics, 91, 66-67. https://doi.org/10.1002/(SICI)1096-8628(20000306)91:1<66::AID-AJMG12>3.0.CO; $\underline{2-\mathrm{P}}$

[5] Braverman, I.M., Keh, A. and Jacobson, B.S. (1990) Ultrastructure and Three-Dimensional Organization of the Telangiectases of Hereditary Hemorrhagic Telangiectasia. Journal of Investigative Dermatology, 95, 422-427. https://doi.org/10.1111/1523-1747.ep12555569

[6] Haitjema, T., Disch, F., Overtoom, T.T., Westermann, C.J. and Lammers, J.W. (1995) Screening Family Members of Patients with Hereditary Hemorrhagic Telangiectasia. The American Journal of Medicine, 99, 519-524. https://doi.org/10.1016/S0002-9343(99)80229-0

[7] Kjeldsen, A.D., Oxhoj, H., Andersen, P.E., Elle, B., Jacobsen, J.P. and Vase, P. (1999) Pulmonary Arteriovenous Malformations: Screening Procedures and Pulmonary Angiography in Patients with Hereditary Hemorrhagic Telangiectasia. Chest, 116, 432-439. https://doi.org/10.1378/chest.116.2.432

[8] Assar, O., Friedman, C.M. and White, R.I. (1991) The Natural History of Epistaxis in Hereditary Hemorrhagic Telangiectasia. Laryngoscope, 101, 977-980.

https://doi.org/10.1288/00005537-199109000-00008

[9] Plauchu, H., De Chadarevian, J.P., Bideau, A. and Robert, J.M. (1999) Age-Related Clinical Profile of Hereditary Hemorrhagic Telangiectasia in an Epidemiologically Recruited Population. American Journal of Medical Geneticist, 32, 291-297. https://doi.org/10.1002/ajmg.1320320302

[10] Desiate, A., Cantore, S., Tullo, D., Profeta, G., Grassi, F.R. and Ballini, A. (2009) 980 $\mathrm{nm}$ Diode Lasers in Oral and Facial Practice: Current State of the Science and Art. International Journal of Medical Sciences, 6, 358-364. https://doi.org/10.7150/ijms.6.358

[11] Hoag, J.B., Terry, P., Mitchell, S., Reh, D. and Merlo, C.A. (2010) An Epistaxis Se- 
verity Score for Hereditary Hemorrhagic Telangiectasia. The Laryngoscope, 120, 838-843. https://doi.org/10.1002/lary.20818

[12] Sabba, C., Gallitelli, M. and Palasciano, G. (2001) Efficacy of Unusually High Doses of Tranexamic Acid for the Treatment of Epistaxis in Hereditary Hemorrhagic Telangiectasia. The New England Journal of Medicine, 345, 926. https://doi.org/10.1056/NEJM200109203451216

[13] Yaniv, E., Preis, M., Hadar, T., Shvero, J. and Haddad, M. (2009) Antiestrogen Therapy for Hereditary Hemorrhagic Telangiectasia: A Double-Blind Placebo-Controlled Clinical Trial. Laryngoscope, 119, 284-288. https://doi.org/10.1002/lary.20065

[14] Pau, H., Carney, A.S. and Murty, G.E. (2001) Hereditary Haemorrhagic Telangiectasia (Osler-Weber-Rendu Syndrome): Otorhinolaryngological Manifestations. Clinical Otolaryngology and Allied Sciences, 26, 93-98.

https://doi.org/10.1046/j.1365-2273.2001.00442.x

[15] Sabba, C., Pasculli, G., Cirulli, A., et al. (2002) Hereditary Hemorrhagic Teleangiectasia (Rendu-Osler-Weber Disease). Minerva Cardioangiologica, 50, 221-238.

[16] Fiorella, M.L., Ross, D.A., White, R.I., et al. (2004) Hereditary Haemorrhagic Telangiectasia: State of the Art. ACTA Otorhinolaryngologica Italica, 24, 330-336.

[17] Kuan, E.C., Peng, K.A., Thompson, C.F., Suh, J.D. and Wang, M.B. (2017) Sinonasal Quality of Life Outcomes Following Laser Treatment of Epistaxis Related to Hereditary Hemorrhagic Telangiectasia. Lasers in Medical Science, 32, 527-531. https://doi.org/10.1007/s10103-017-2144-7

[18] Levy, J.L. and Berwald, C. (2004) Treatment of Vascular Abnormalities with a Long-Pulse Diode at $980 \mathrm{~nm}$. Journal of Cosmetic and Laser Therapy, 6, 217-221. https://doi.org/10.1080/14764170410003020

[19] Kunishige, J.H., Goldberg, L.H. and Friedman, P.M. (2007) Laser Therapy for Leg Veins. Clinics in Dermatology, 25, 454-461. https://doi.org/10.1016/j.clindermatol.2007.05.008

[20] Karapantzos, I., Tsimpiris, N., Goulis, D.G., Van Hoecke, H., Van Cauwenberge, P. and Danielides, V. (2005) Management of Epistaxis in Hereditary Hemorrhagic Telangiectasia by Nd:YAG Laser and Quality of Life Assessment Using the HR-QoL Questionnaire. European Archives of Oto-Rhino-Laryngology, 262, 830-833. https://doi.org/10.1007/s00405-004-0911-0

[21] Jorgensen, G., Lange, B., Wanscher, J.H. and Kjeldsen, A.D. (2011) Efficiency of Laser Treatment in Patients with Hereditary Hemorrhagic Telangiectasia. European Archives of Oto-Rhino-Laryngology, 268, 1765-1770.

https://doi.org/10.1007/s00405-011-1677-9 
Appendix-Epistaxis Severiy Score (ESS)

How often do you TYPICALLY have nose bleeding? (coefficient 0.14)

0 - Less than monthly 3 - Several per week

1 - Once per month 4 - Once per day

2 - Once per week 5 - Several each day

How long do your TYPICAL nose bleeds last? (coefficient 0.25 )

$0-<1$ minute $\quad 3-16-30$ minutes

$1-1-5$ minutes $\quad 4->30$ minutes

$2-6-15$ minutes

How would you describe your TYPICAL nose bleeding intensity? (coefficient 0.25)

$0-$ Not typically gushing 1 - Typically gushing or pouring Have you every sought medical attention for nose bleeding? (coefficient 0.30)
0 - No
1 - Yes

Are you anemic (low blood count) currently? (coefficient 0.20)
$0-$ No
1 - Yes

Have you ever received a red blood cell transfusion specifically because of nose bleeding? (coefficient 0.31 )

$0-$ No 1 - Yes

Six questions are answered, the number of the response is multiplied by the respective coefficient, and the sum of these gives the raw epistaxis severity score. 